\title{
Korean Red Ginseng and Portulaca oleracea Extracts Attenuate Lipopolysaccharide-induced Inflammation via Downregulation of Nuclear Factor Kappa-B and the Mitogen-activated Protein Kinase Signaling Pathway in Macrophage Cell Line RAW 264.7
}

\author{
H M Arif Ullah ${ }^{1, \$, *}$, Tae-Hwan Kim ${ }^{1, \$, * *}$, Evelyn Saba ${ }^{2, * *}$, \\ Sung Dae Kim ${ }^{1, * *}$ and Man Hee Rhee ${ }^{1, \hat{*}, * *}$ \\ ${ }^{1}$ Department of Veterinary Medicine, College of Veterinary Medicine, \\ Kyungpook National University, Daegu 41566, Korea \\ ${ }^{2}$ Department of Veterinary Biomedical Sciences, Faculty of Veterinary and Animal Sciences, \\ Pir Mehr Ali Shah-Arid Agriculture University, Rawalpindi, Pakistan
}

\begin{abstract}
Korean red ginseng (Panax ginseng Meyer) is a well-known traditional medicine, with numerous biological functions in the body. Portulaca oleracea ( $P$. ole) belongs to the Portulacaceae family and has bioactive potential as a traditional medicine. This study aimed to determine the anti-inflammatory effects of Korean red ginseng extract (RGE) and $P$. ole extract on lipopolysaccharide (LPS)-treated RAW 264.7 cells. The combination of RGE $(50 \mu \mathrm{g} / \mathrm{mL})$ and P. ole (6.25 $\mu \mathrm{g} /$ $\mathrm{mL}$ ) extracts significantly suppressed LPS-induced nitric oxide synthesis. The expression of proinflammatory mediators, including inducible nitric oxide synthase (iNOS) and cyclooxygenase-2 (COX-2), and proinflammatory cytokines, including interleukin- $1 \beta$, interleukin- 6 , and tumor necrosis factor- $\alpha$, were markedly decreased by the combined treatment with RGE $(50 \mu \mathrm{g} / \mathrm{mL})$ and P. ole $(6.25 \mu \mathrm{g} / \mathrm{mL})$. Moreover, iNOS and COX-2 protein expression levels were also significantly reduced in the combined treatment compared to the LPS-stimulated group. In addition, the nuclear translocation of phosphorylated nuclear factor kappa-B was suppressed by the treatment with RGE and $P$. ole. Moreover, the mitogenactivated protein kinase pathway was also partially inhibited by the combination treatment with RGE and $P$. ole. Our results demonstrate that the treatment mixture with RGE and $P$. ole could be used as functional food and therapeutic herbal medicine in various inflammatory diseases.
\end{abstract}

Key Words: Korean red ginseng, Portulaca oleracea, Functional food, Anti-inflammatory effects, RAW 264.7 cells

\section{INTRODUCTION}

Ginseng (Panax ginseng Meyer) is an ancient herbal remedy that is used widely for its promising pharmaco- logical properties on overall health (Saba et al., 2018; Saba et al., 2020b). As a traditional and alternative therapy, red ginseng extract (RGE) has been used to treat many diseases for over two thousand years (Wee et al., 2011). Ginsenosides are the major bioactive ginseng constituents which are essen-

Received: April 30, 2021 / Revised: May 26, 2021 / Accepted: June 4, 2021

*Graduate student, ${ }^{* *}$ Professor.

$\S$ These authors equally contributed to this work.

${ }^{\dagger}$ Corresponding author: Man Hee Rhee. Department of Veterinary Medicine, College of Veterinary Medicine, Kyungpook National University, Daegu 41566, Korea.

Tel: +82-53-950-5967, Fax: +82-53-950-5955, e-mail: rheemh@knu.ac.kr

(C) The Korean Society for Biomedical Laboratory Sciences. All rights reserved.

(c) This is an Open Access article distributed under the terms of the Creative Commons Attribution Non-Commercial License (http://creativecommons.org/licenses/by-nc/3.0/) which permits unrestricted non-commercial use, distribution, and reproduction in any medium, provided the original work is properly cited. 
tial for its therapeutic effects (Farzaei et al., 2015; Saba et al., 2020a).

Portulaca oleracea (P. ole), belonging to the Portulacaceae family, is distributed worldwide. $P$. ole is considered as a medicinal food, especially in China (Yen et al., 2001; Li et al., 2016). Therefore, $P$. ole is widely used as a vegetable crop due to its many medicinal properties, including antibacterial, antioxidant, anti-inflammatory, and antiaging effects (Chan et al., 2000; Chen et al., 2012; E Abdel Moneim, 2013; Zhou et al., 2015; Baradaran Rahimi et al., 2019).

Inflammation is a critical part of the immune system that protects the host from harmful stimuli (Pyee et al., 2014; Ullah et al., 2014). However, dysregulated inflammation is closely related with many chronic diseases. Hence, inflammatory response regulation remains a challenge. Macrophages play key role in the operation of inflammatory processes, mainly through the production of various inflammatory factors, such as nitric oxide (NO), inducible nitric oxide synthase (iNOS), cyclooxygenase-2 (COX-2), interleukin$1 \beta$ (IL-1 $\beta$ ), interleukin-6 (IL-6), and tumor necrosis factor$\alpha$ (TNF)- $\alpha$ (Choi et al., 2014). Thus, the inhibition of macrophage activation is considered a vital approach to treat inflammatory diseases.

In this study, we selected the two extracts because combination of RGE $(50 \mu \mathrm{g} / \mathrm{mL})$ and $P$. ole $(6.25 \mu \mathrm{g} / \mathrm{mL})$ showed higher effect than separate effect. This combined treatment exhibited synergistic effect in RAW cells without cytotoxicity. In order to show synergism, weak inhibitory of both extract (Korean red ginseng and Portulaca oleracea) was used. We investigated the anti-inflammatory effects of Korean RGE and $P$. ole extract on lipopolysaccharide (LPS)-induced RAW 264.7 cells, using NO and cell viability assays and mRNA and protein expression of proinflammatory mediators and cytokines. Thus, our study demonstrated that RGE and P. ole extracts have potential anti-inflammatory properties in the regulation of inflammation and may be used as adjuvant therapy in the treatment of various inflammatory diseases.

\section{MATERIALS AND METHODS}

\section{Chemicals and reagents}

Dulbecco's modified eagle medium (DMEM), fetal bovine serum (FBS), streptomycin, and penicillin were purchased from WelGene, Daegu, Republic of Korea. LPS and MTT were obtained (Sigma, St. Louis MO, USA). TRIzol was obtained from Invitrogen (Carsbad, CA, USA). Polymerase chain reaction (PCR) primers were obtained from Bioneer (Daejeon, Republic of Korea). Antibodies for iNOS, COX2, IRAK1, phospho (p)-TAK1, p-IKK $\alpha / \beta$, p-IкB/ $\alpha, p-N F-\kappa B$, p-JNK, total (T)-JNK, p-P38, T-P38, p-ERK, T-ERK, and $\beta$-actin were purchased (Cell Signaling Technology, USA).

\section{Preparation of the Korean red ginseng and Portulaca oleracea extracts}

We purchased Korean red ginseng and Portulaca oleracea from the company and ground them separately into a fine coarse powder. We then extracted separately in $70 \%$ ethanol using a heating mantle at $80^{\circ} \mathrm{C}$ for 2 hours, followed by extract condensation with the rotary evaporator system. The crude extract was frozen at $-70^{\circ} \mathrm{C}$ overnight and lyophilized using a freeze dryer to make a dry fine powder. Finally, the powder was dissolved in DMSO at the time of the experiment.

\section{Cell culture and treatment}

The macrophage cell line RAW 264.7 was cultured in DMEM supplemented with heat-inactivated $10 \%$ FBS, penicillin (100 units/mL), and streptomycin $(100 \mu \mathrm{g} / \mathrm{mL}, 1 \%$ antibiotics). Then, cells were incubated in a humidified incubator at $37^{\circ} \mathrm{C}$ with $5 \% \mathrm{CO}_{2}$.

\section{Nitric oxide and cell viability assay}

RAW 264.7 cells were seeded $\left(2 \times 10^{5}\right)$ per $\mathrm{mL}$ in 24 well plates. Cells were treated after $24 \mathrm{~h}$ with different concentrations of RGE $(50 \mu \mathrm{g} / \mathrm{mL})$ and/or P. ole $(6.25 \mu \mathrm{g}$ $/ \mathrm{mL}$ ), followed by $0.1 \mu \mathrm{g} / \mathrm{mL}$ of LPS after $30 \mathrm{~min}$. Cells were incubated for $18 \mathrm{~h}$ for NO induction. Supernatant was collected for NO analysis using the Griess reagent and read at $540 \mathrm{~nm}$ using a microplate reader. For the measurement of cell viability, the MTT reagent was added to the cells and incubated for $2 \sim 3 \mathrm{~h}$, and dimethyl sulfoxide was used to dissolve the crystal. The dissolved solution was analyzed at $560 \mathrm{~nm}$ using a microplate reader. 


\section{Polymerase chain reaction}

RAW 264.7 cells were seeded $\left(5 \times 10^{5}\right)$ per $\mathrm{mL}$ in 6 well plates. After $24 \mathrm{~h}$, the cells were treated with different concentrations of RGE $(50 \mu \mathrm{g} / \mathrm{mL})$ and/or P. ole $(6.25 \mu \mathrm{g}$ $/ \mathrm{mL})$, followed by LPS $(0.1 \mu \mathrm{g} / \mathrm{mL})$ after $30 \mathrm{~min}$. Then, cells were incubated for another $18 \mathrm{~h}$. Using the TRIzol reagent, RNA was extracted and measured using a nanophotometer. Kits were used according to the manufacturer's protocol (Bioneer, Daejeon, Republic of Korea) for reverse transcription. The cDNA product was subjected to PCR using gel electrophoresis, and gene expression was normalized using GAPDH.

\section{Western blot analysis}

The western blot analysis was done as described previously (Ullah et al., 2021). Cells were seeded $\left(5 \times 10^{5}\right.$ per $\mathrm{mL})$ in 6-well plates. After $24 \mathrm{~h}$, the cells were treated with RGE $(50 \mu \mathrm{g} / \mathrm{mL})$ and/or P. ole $(6.25 \mu \mathrm{g} / \mathrm{mL})$, followed by LPS $(0.1 \mu \mathrm{g} / \mathrm{mL})$ after $30 \mathrm{~min}$. The cells were then incubated for another $18 \mathrm{~h}$. Using Pro-Prep solution, protein was extracted, and the protein concentration was measured using the Bradford method. Proteins were separated in 10\% SDSPAGE and transferred into PVDF membrane. A 5\% skim milk solution was used as a blocking buffer for $1 \mathrm{~h}$. Primary antibodies $(1: 1,000)$ were incubated overnight, and a secondary antibody $(1: 3,000)$ was used for $1 \mathrm{~h}$. The membrane was developed in a developer, and protein bands were analyzed using ImageJ.

\section{Statistical analysis}

Data were represented as the mean \pm standard error of the mean. Significant differences were analyzed by one-way analysis of variance. Statistical significance was set at $* P<$ $0.05, * * P<0.01$, and $* * * P<0.001$ compared to the LPStreated group.

\section{RESULTS}

\section{Effects of RGE and $P$. ole extracts on LPS-induced NO production and cell viability}

First, to confirm LPS-induced NO production in the raw macrophage cell line, we measured the NO assay. Our results showed that the LPS-stimulated group significantly produced more NO compared to the basal group, but RGE and $P$. ole extract individually inhibited NO production. Of note, the combined use of RGE $(50 \mu \mathrm{g} / \mathrm{mL})$ and $P$. ole $(6.25 \mu \mathrm{g} / \mathrm{mL}$ ) significantly suppressed NO production (Fig. 1A). Altogether, this result suggests that combination treatment with RGE and $P$. ole has anti-inflammatory properties.
A

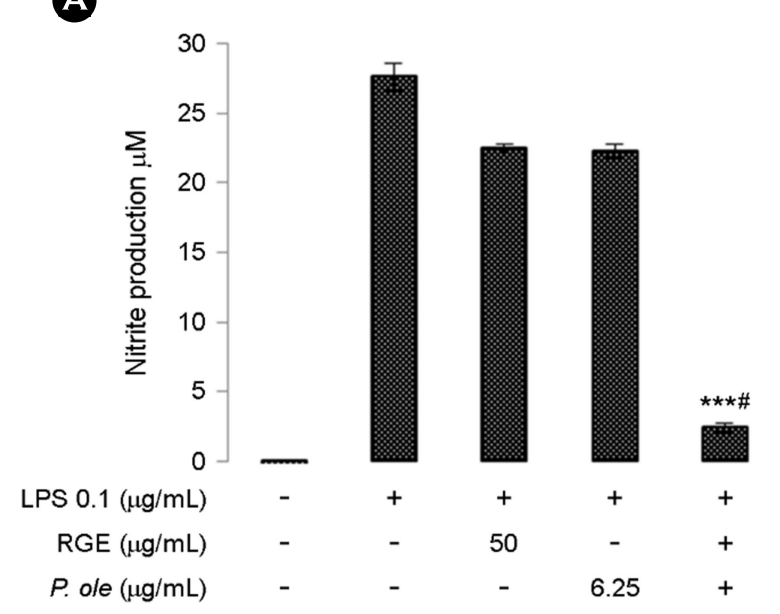

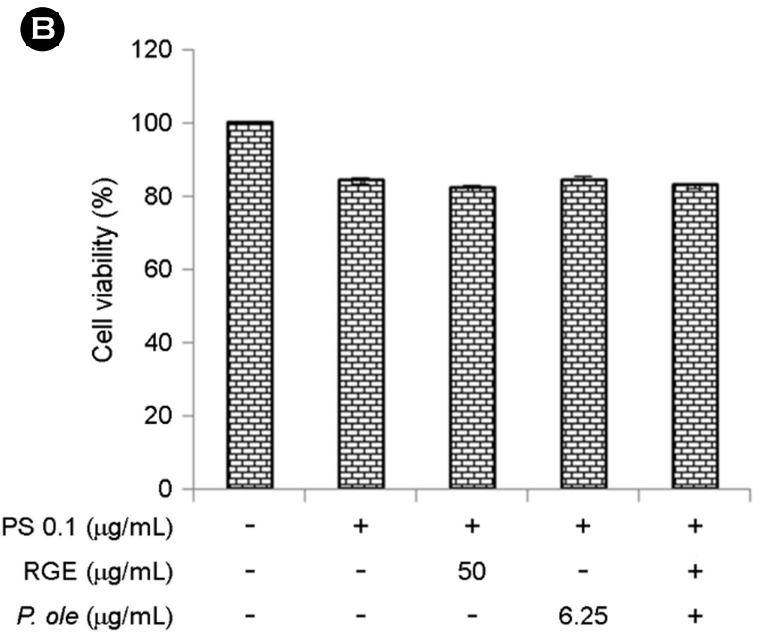

Fig. 1. Effects of Korean red ginseng extract (RGE) and Portulaca oleracea (P. ole) extract on nitric oxide (NO) production and cell viability in macrophage RAW 264.7 cells. (A) Suppression of NO production in the RAW 264.7 cells pretreated with the RGE $(50 \mu \mathrm{g} / \mathrm{mL})$ and $P$. ole $(6.25 \mu \mathrm{g} / \mathrm{mL})$ extract for $30 \mathrm{~min}$, followed by cotreatment with LPS $(0.1 \mu \mathrm{g} / \mathrm{mL})$ for $18 \mathrm{~h}$. (B) Cell viability of RAW 264.7 cells treated with RGE $(50 \mu \mathrm{g} / \mathrm{mL})$ and $P$. ole $(6.25 \mu \mathrm{g} / \mathrm{mL})$. ${ }^{* * *} P<0.001$ combination of RGE and $P$. ole against individual treatment. 
A

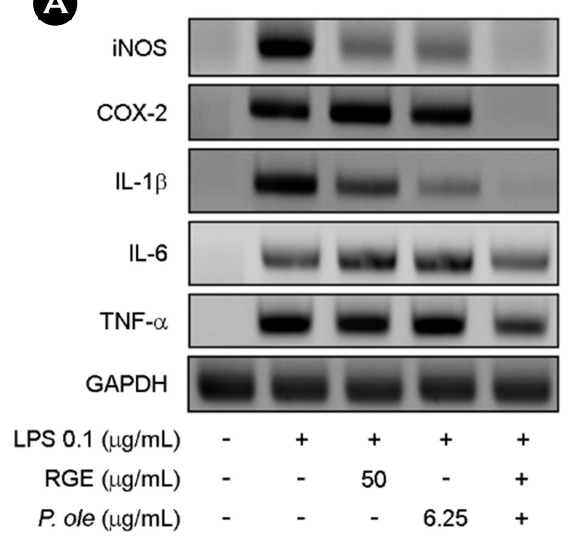

B

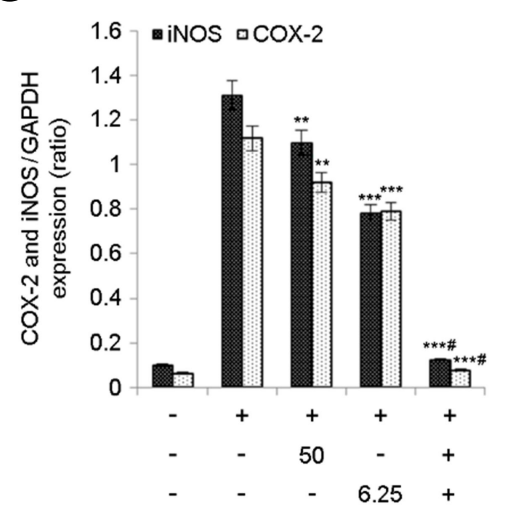

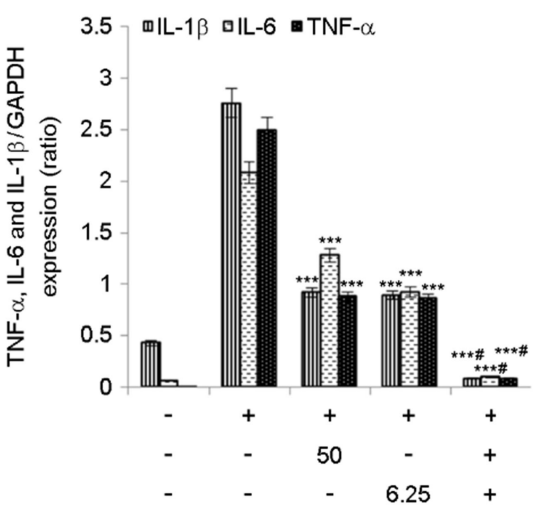

Fig. 2. Effects of Korean red ginseng extract (RGE) and Portulaca oleracea (P. ole) extracts on lipopolysaccharide (LPS)-induced proinflammatory mediators and cytokines in macrophage RAW 264.7 cells. (A) mRNA expression of iNOS, COX2, IL-1 $\beta$, IL-6, and TNF- $\alpha$. GAPDH was used as a housekeeping gene. (B) Quantitation of polymerase chain reaction (PCR) analysis of iNOS, COX2, IL-1 $\beta$, IL-6, and TNF- $\alpha$. ${ }^{* *} P<0.01,{ }^{* * *} P<0.001$, and ${ }^{* * * *} P<0.001$ combination of RGE and $P$. ole against individual treatment.

The cell viability assay determined the absence of cytotoxic effects of the RGE and $P$. ole extracts on the raw macrophage cells. We chose the concentration of RGE $(50 \mu \mathrm{g} / \mathrm{mL})$ and $P$. ole $(6.25 \mu \mathrm{g} / \mathrm{mL})$ based on the cytotoxic effects (Fig. 1B). Thus, the cell viability assay suggests that RGE and $P$. ole have no cytotoxic effects on the concentrations used (50 and $6.25 \mu \mathrm{g} / \mathrm{mL}$, respectively).

Inhibitory effects of RGE and $P$. ole extracts on LPSinduced proinflammatory mediators and cytokines in macrophages

PCR analysis was used to determine the effects of RGE and $P$. ole extracts on mRNA expression of proinflammatory mediators and cytokines in the LPS-treated macrophage cell line RAW 264.7. PCR results indicated that iNOS, COX2, IL-1 $\beta$, IL-6, and TNF- $\alpha$, were significantly upregulated in the LPS-stimulated treated group compared with the basal group. However, treatment with RGE $(50 \mu \mathrm{g} / \mathrm{mL})$ and $P$. ole $(6.25 \mu \mathrm{g} / \mathrm{mL})$ extracts markedly suppressed the release of inflammatory mediators and cytokines (Fig. 2A and B). Overall, these results indicate that LPS-induced inflammatory mediator secretion was inhibited by RGE and P. ole.

Effects of RGE and $P$. ole extracts on the protein expression of iNOS and COX2

Western blot analysis was used to confirm the secretion of proinflammatory mediators suppressed by RGE and $P$. ole. The Western blot results revealed that inflammatory mediators, including iNOS and COX2, were significantly upregulated in the LPS-stimulated group compared to control group, suggesting that LPS successfully induced inflammation. However, treatment with RGE $(50 \mu \mathrm{g} / \mathrm{mL})$ and $P$. ole $(6.25 \mu \mathrm{g} / \mathrm{mL})$ extracts significantly decreased the expression of iNOS and COX2 levels compared to the LPS-treated group (Fig. 3A and B). Thus, the immunoblot results suggest that RGE $(50 \mu \mathrm{g} / \mathrm{mL})$ and $P$. ole $(6.25 \mu \mathrm{g} / \mathrm{mL})$ extracts have significant anti-inflammatory activities in protein expression levels.

\section{RGE and $P$. ole prevent the activation of NF-кB signaling in LPS-induced cells}

The NF- $\mathrm{BB}$-signaling pathway was investigated to clarify the anti-inflammatory mechanism of RGE and $P$. ole. The protein expression levels of IRAK-1, p-TAK-1, p-IKK $\alpha / \beta$, $\mathrm{p}-\mathrm{IkB} / \alpha$, and $\mathrm{p}-\mathrm{NF}-\mathrm{\kappa} B$ were analyzed by Western blotting, whereas $\beta$-actin was used as an internal standard. Phosphorylation of NF- $\kappa \mathrm{B}$ was increased in the LPS-stimulated group, whereas $\mathrm{p}-\mathrm{NF}-\kappa \mathrm{B}$ was significantly downregulated by the combination of RGE $(50 \mu \mathrm{g} / \mathrm{mL})$ and $P$. ole $(6.25 \mu \mathrm{g}$ $/ \mathrm{mL}$ ) extracts (Fig. 4A and B). Overall, these results indicate that RGE and $P$. ole exhibited anti-inflammatory effects by blocking the activation of the NF- $\mathrm{KB}$ pathway. 



Fig. 3. Effects of Korean red ginseng extract (RGE) and Portulaca oleracea $(P$. ole) extracts on inflammatory mediators related protein such as iNOS and COX2 in macrophage RAW 264.7 cells. (A) Western blot analysis of iNOS and COX2. RGE, P. ole, and lipopolysaccharide (LPS) were used $(50 \mu \mathrm{g} / \mathrm{mL}, 6.25 \mu \mathrm{g} / \mathrm{mL}$, and $0.1 \mu \mathrm{g} / \mathrm{mL}$, respectively). $\beta$-actin was used as a normalizing protein. (B) Quantitation of western blot protein band of iNOS and COX2. ${ }^{* * *} P<0.001$, and ${ }^{* * * *} P<0.001$ combination of RGE and $P$. ole against individual treatment.

A



B

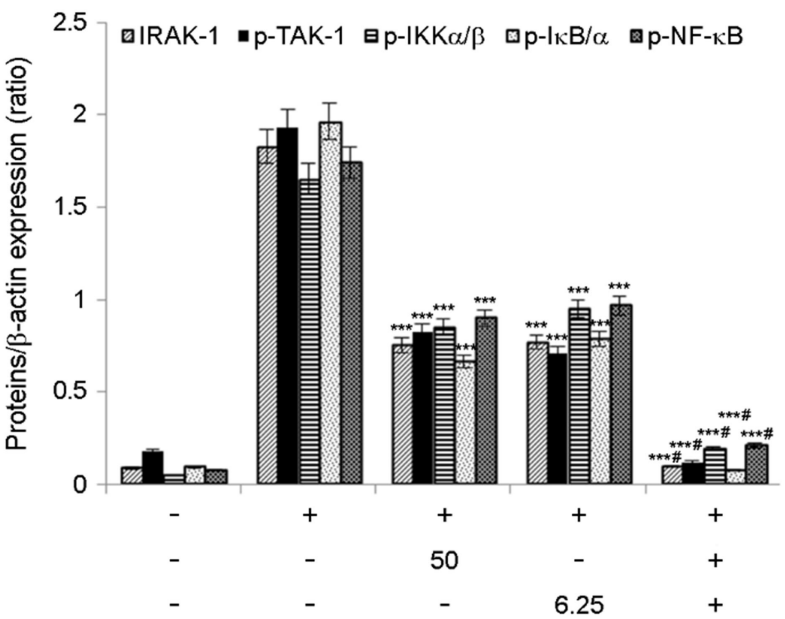

Fig. 4. Effects of Korean red ginseng extract (RGE) and Portulaca oleracea (P. ole) on the activation of the NF-KB signaling pathway


an internal standard. (B) Quantitation of western blot protein band. ${ }^{* * *} P<0.001$, and ${ }^{* * * *} P<0.001$ combination of RGE and $P$. ole against individual treatment.

RGE and $P$. ole prevent the activation of MAPK signaling in LPS-induced cells

The mitogen-activated protein kinase (MAPK) pathway was investigated to understand the other anti-inflammatory mechanisms of RGE and $P$. ole. The expression levels of p-P38, T-P38, p-JNK, T-JNK, p-ERK, and T-ERK proteins were analyzed by Western blotting, with the $\beta$-actin antibody used as an internal standard. The production of phosphoryl- ated (p)-JNK, p-P38, and p-ERK were markedly increased in the LPS-stimulated group, whereas treatment with RGE $(50 \mu \mathrm{g} / \mathrm{mL})$ and $P$. ole $(6.25 \mu \mathrm{g} / \mathrm{mL})$ extracts significantly inhibited the MAPK pathway-related protein expressions (Fig. 5A and B). Collectively, Western blot results suggest that RGE and $P$. ole showed anti-inflammatory effects by inhibiting the MAPK pathway. 

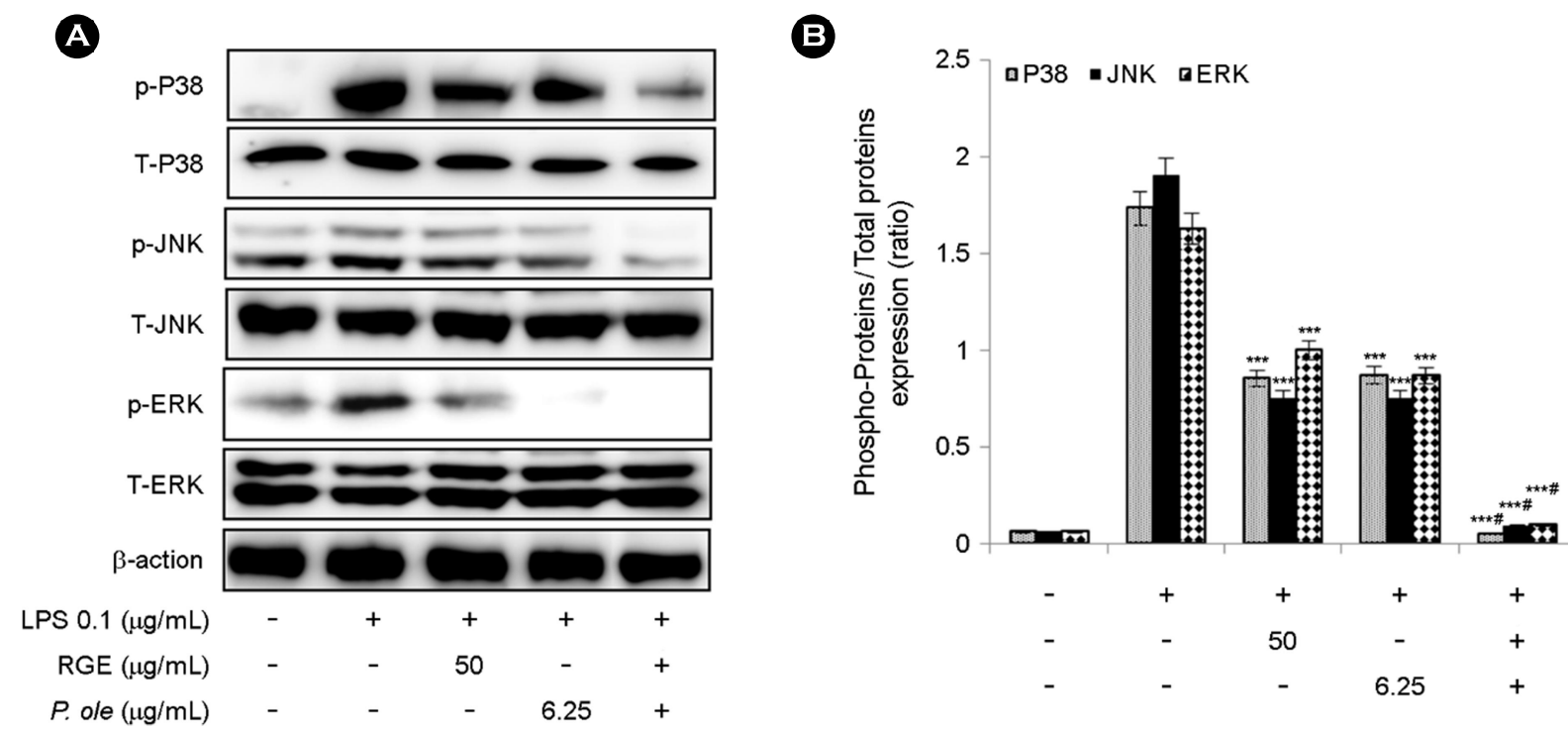

Fig. 5. Korean red ginseng extract (RGE) and Portulaca oleracea $(P$. ole) prevent the activation of MAPK signaling pathway in macrophage RAW 264.7 cells. (A) Western blot analysis of MAPK pathways including p-P38, T-P38, p-JNK. T-JNK, p-ERK, and T-ERK. $\beta$-actin was used as an internal standard. (B) Quantitation of western blot protein band. ${ }^{* * *} P<0.001$, and ${ }^{* * * \#} P<0.001$ combination of RGE and $P$. ole against individual treatment.

\section{DISCUSSION}

Inflammation is an important biological immune response initiated by harmful stimuli and is closely associated with a number of inflammatory diseases, such as atherosclerosis, Alzheimer's disease, cancers, diabetes mellitus, and rheumatoid arthritis (Bag-Ozbek and Giles, 2015; Bessueille and Magne, 2015; Ullah et al., 2021). In this study, lipopolysaccharide (LPS)-treated RAW 264.7 macrophage cells were used as the experimental model. Macrophages easily respond to LPS, the bacterial endotoxin, which is the key stimulant of inflammation (Miao et al., 2019). Various proinflammatory mediators and cytokines, including NO, inducible nitric oxide synthase (iNOS), cyclooxygenase-2 (COX-2), interleukin$1 \beta$ (IL-1 $\beta$ ), interleukin-6 (IL-6), and tumor necrosis factor- $\alpha$ (TNF)- $\alpha$, are released by activated macrophages to regulate the immune system against patho- gens.

The extreme production of NO related to iNOS synthesis is involved in the inflammatory process. iNOS plays a vital role in releasing NO during the pathophysiology of inflammatory diseases (Vodovotz et al., 1993; Zamora et al., 2000; Li et al., 2016). Additionally, COX2 is also stimulated by inflammatory stimuli during the inflammatory response. In our study, only the LPS-stimulated group showed overexpression of NO production compared to the basal group, whereas pretreatment with RGE and $P$. ole extracts reduced NO production. In particular, the combined treatment of RGE $(50 \mu \mathrm{g} / \mathrm{mL})$ and $P$. ole $(6.25 \mu \mathrm{g} / \mathrm{mL})$ significantly decreased NO induction (Fig. 1A).

The mRNA expression levels of proinflammatory mediators, such as iNOS and COX2, and inflammatory cytokines, including IL-1 $\beta$, IL-6, and TNF- $\alpha$, were upregulated in the LPS-treated group, whereas the combination treatment of RGE and $P$. ole significantly downregulated mRNA levels of proinflammatory factors (Fig. 2). These results suggested that the mixture of RGE and $P$. ole has the potential to inhibit proinflammatory factors. Previously, it was found that protein expression levels of iNOS and COX2 were increased in LPS-induced RAW 264.7 cells (Li et al., 2016; Miao et al., 2019). Our results also support the previous findings that iNOS and COX2 were upregulated in the LPS-stimulated group and that treatment with RGE $(50 \mu \mathrm{g} / \mathrm{mL})$ and $P$. ole $(6.25 \mu \mathrm{g} / \mathrm{mL})$ significantly reduced the protein expression of iNOS and COX2 (Fig. 3).

It was reported that NF- $\kappa \mathrm{B}$ and MAPK signaling pathways are the key pathways for the inflammatory process 
(Chen et al., 2003; Miao et al., 2019). Therefore, we explored the antiinflammatory mechanism of the RGE and $P$. ole extracts in the macrophage cell line. Protein expression levels of IRAK1, p-TAK1, p-IKK $\alpha / \beta, p-I \kappa B / \alpha, p-N F-\kappa B, p-J N K$, T-JNK, p-P38, T-P38, p-ERK, and T-ERK were investigated using Western blot. For the macrophage RAW 264.7 cell line treated with LPS, the NF- $\kappa$ B and MAPK signaling pathways were increased by LPS treatment, whereas treatment with RGE $(50 \mu \mathrm{g} / \mathrm{mL})$ and $P$. ole $(6.25 \mu \mathrm{g} / \mathrm{mL})$ significantly suppressed the NF- $\kappa$ B and MAPK signaling proteins (Fig. 4 and 5). These findings suggest that RGE and $P$. ole inhibited the LPS-stimulated inflammation by blocking the NF- $\mathrm{KB}$ and MAPK signaling pathways.

In conclusion, this study demonstrated that RGE and $P$. ole extract have potential anti-inflammatory effects. The combination of RGE and $P$. ole could be used as a functional food along with modern treatments in inflammatory diseases

\section{ACKNOWLEDGMENT}

This study was funded by the National Research Foundation of Korea (2018R1D1A1A09083797). The authors would like to thank Professor Man Hee Rhee for his supervision and support, H M Arif Ullah for the manuscript writing and final review and editing and Yuan Yee Lee for technical support.

\section{CONFLICT OF INTEREST}

The authors have declared no conflict of interest.

\section{REFERENCES}

Bag-Ozbek A, Giles JT. Inflammation, adiposity, and atherogenic dyslipidemia in rheumatoid arthritis: Is there a paradoxical relationship? Current Allergy and Asthma Rreports. 2015. 15: 497.

Baradaran Rahimi V, Rakhshandeh H, Raucci F, Buono B, Shirazinia R, Samzadeh Kermani A, Maione F, Mascolo N, Askari VR. Anti-inflammatory and anti-oxidant activity of Portulaca oleracea extract on lps-induced rat lung injury. Molecules. 2019. 24: 139.

Bessueille L, Magne D. Inflammation: A culprit for vascular calcification in atherosclerosis and diabetes. Cellular and Molecular
Life Science. 2015. 72: 2475-2489.

Chan K, Islam M, Kamil M, Radhakrishnan R, Zakaria M, Habibullah M, Attas A. The analgesic and anti-inflammatory effects of Portulaca oleracea 1. Subsp. Sativa (haw.) celak. Journal of Ethnopharmacology. 2000. 73: 445-451.

Chen B, Zhou H, Zhao W, Zhou W, Yuan Q, Yang G. Effects of aqueous extract of Portulaca oleracea 1. On oxidative stress and liver, spleen leptin, par $\alpha$ and fas mrna expression in highfat diet induced mice. Molecular Biology Reports. 2012. 39: 7981-7988.

Chen YH, Layne MD, Chung SW, Ejima K, Baron RM, Yet SF, Perrella MA. Elk-3 is a transcriptional repressor of nitricoxide synthase 2. Journal of Biological Chemistry. 2003. 278 39572-39577.

Choi WS, Jeong JW, Kim SO, Kim GY, Kim BW, Kim CM, Seo YB, Kim WY, Lee SY, Jo KHJ. Anti-inflammatory potential of peat moss extracts in lipopolysaccharide-stimulated raw 264.7 macrophages. International Journal of Molecular Medicine. 2014. 34: 1101-1109.

E Abdel Moneim A. The neuroprotective effects of purslane (Portulaca oleracea) on rotenone-induced biochemical changes and apoptosis in brain of rat. CNS \& Neurological DisordersDrug Targets. 2013. 12: 830-841.

Farzaei MH, Farzaei F, Gooshe M, Abbasabadi Z, Rezaei N, Abdolghaffari AH. Potentially effective natural drugs in treatment for the most common rheumatic disorder: Osteoarthritis. Rheumatology International. 2015. 35: 799-814.

Li CY, Meng YH, Ying ZM, Xu N, Hao D, Gao MZ, Zhang WJ, Xu L, Gao YC, Ying XX. Three novel alkaloids from Portulaca oleracea 1 . And their anti-inflammatory effects. Journal of Agriculture and Food Chemistry. 2016. 64: 5837-5844.

Miao L, Tao H, Peng Y, Wang S, Zhong Z, El-Seedi H, Dragan S, Zengin G, San Cheang W, Wang YJ. The anti-inflammatory potential of Portulaca oleracea 1. (purslane) extract by partial suppression on nf-kb and mapk activation. Food Chemistry. 2019. 290: 239-245

Pyee Y, Chung HJ, Choi TJ, Park HJ, Hong JY, Kim JS, Kang SS Lee SK. Suppression of inflammatory responses by handelin, a guaianolide dimer from chrysanthemum boreale, via downregulation of $\mathrm{nf}-\mathrm{\kappa b}$ signaling and pro-inflammatory cytokine production. Journal of Natural Products. 2014. 77: 917-924.

Saba E, Jeong D, Irfan M, Lee YY, Park SJ, Park CK, Rhee MH. Anti-inflammatory activity of rg3-enriched korean red ginseng extract in murine model of sepsis. Evidence-Based Comple- 
mentary and Alternative Medicine. 2018. 2018: 6874692.

Saba E, Kim SH, Lee YY, Park CK, Oh JW, Kim TH, Kim HK, Roh SS, Rhee MH. Korean red ginseng extract ameliorates melanogenesis in humans and induces antiphotoaging effects in ultraviolet b-irradiated hairless mice. Journal of Ginseng Research. 2020a. 44: 496-505.

Saba E, Lee YY, Kim M, Hyun SH, Park CK, Son E, Kim DS, Kim $\mathrm{SD}$, Rhee MH. A novel herbal formulation consisting of red ginseng extract and epimedium koreanum nakai-attenuated dextran sulfate sodium-induced colitis in mice. Journal of Ginseng Research. 2020b. 44: 833-842.

Ullah H, Elfadl A, Park S, Kim YD, Chung MJ, Son JY, Yun HH, Park JM, Yim JH, Jung SJ. Nogo-a is critical for proinflammatory gene regulation in myocytes and macrophages. Cells. 2021. 10: 282.

Ullah HA, Zaman S, Juhara F, Akter L, Tareq SM, Masum EH, Bhattacharjee R. Evaluation of antinociceptive, in-vivo \& invitro anti-inflammatory activity of ethanolic extract of curcuma zedoaria rhizome. BMC Complementary and Alternative Medicine. 2014. 14: 1-12.

Vodovotz Y, Bogdan C, Paik J, Xie Q, Nathan C. Mechanisms of suppression of macrophage nitric oxide release by transforming growth factor beta. The Journal of Experimental Medicine. 1993. 178: 605-613.
Wee JJ, Park KM, Chung AS. Biological activities of ginseng and its application to human health. Herbal Medicine: Biomolecular and Clinical Aspects. $2^{\text {nd }}$ edition. 2011.

Yen G, Chen H, Peng HH. Evaluation of the cytotoxicity, mutagenicity and antimutagenicity of emerging edible plants. Food and Chemical Toxicology. 2001. 39: 1045-1053.

Zamora R, Vodovotz Y, Billiar TR. Inducible nitric oxide synthase and inflammatory diseases. Molecular Medicine. 2000. 6:347373.

Zhou YX, Xin HL, Rahman K, Wang SJ, Peng C, Zhang H. Portulaca oleracea 1.: A review of phytochemistry and pharmacological effects. BioMed Research International. 2015. 2015: 925631.

https://doi.org/10.15616/BSL.2021.27.2.51

Cite this article as: Ullah H M Arif, Kim TH, Saba E, Kim SD, Rhee MH. Korean Red Ginseng and Portulaca oleracea Extracts Attenuate Lipopolysaccharide-induced Inflammation via Downregulation of Nuclear Factor Kappa-B and the Mitogen-activated Protein Kinase Signaling Pathway in Macrophage Cell Line RAW 264.7. Biomedical Science Letters. 2021. 27: 51-58. 